\title{
Reawakening the cellular death program in neoplasia through the therapeutic blockade of IAP function
}

\author{
Casey W. Wright ${ }^{1}$ and Colin S. Duckett ${ }^{1,2}$
}

${ }^{1}$ Department of Pathology, and ${ }^{2}$ Department of Internal Medicine, University of Michigan, Ann Arbor, Michigan, USA.

\begin{abstract}
Recent studies have shown that members of the inhibitor of apoptosis (IAP) protein family are highly expressed in several classes of cancer. The primary implication of these findings is that the elevated expression of IAPs is not coincidental but actually participates in oncogenesis by helping to allow the malignant cell to avoid apoptotic cell death. This concept, together with the discovery of several IAP-regulatory proteins that use a conserved mode of action, has stimulated a major effort by many research groups to devise IAP-targeting strategies as a means of developing novel antineoplastic drugs. In this Review, we consider the evidence both for and against the IAPs being valid therapeutic targets, and we describe the types of strategies being used to neutralize their functions.
\end{abstract}

\section{IAPs: structure and function}

Inhibitor of apoptosis (iap) genes were first described in insect viruses through a genetic screen to identify compensatory replacements for the loss of the baculoviral antiapoptotic $\mathrm{p} 35$ protein, which functions to block baculovirus-induced apoptosis during infection (1-3). Since the initial identification in baculoviruses, iap homologs have been identified across phyla from Caenorhabditis elegans and yeast to insects and mammals. As many as 8 human iap gene products have been identified (reviewed in ref. 4), XIAP (hILP/ MIHA/BIRC4), hILP-2 (TS-IAP), cellular IAP1 (c-IAP1/HIAP2/ MIHB/BIRC2), cellular IAP2 (c-IAP2/HIAP1/MIHC/BIRC3), melanoma-associated IAP (ML-IAP/Livin/KIAP1/BIRC7), neuronal apoptosis-inhibitory protein (NAIP/BIRC1), survivin (TIAP1/ BIRC5), and Apollon (BRUCE/BIRC6) (Figure 1A). These IAP proteins have been shown to play, for the most part, nonredundant cellular roles that range from apoptotic inhibition to the formation of the mitotic spindle during cytokinesis. For this reason, the term "IAP" is somewhat misleading, and these factors are often referred to as BIR-containing proteins, or BIRPs (5), a term derived from the presence of what has become the defining motif of this family, an approximately 65-residue domain rich in histidines and cysteines known as the baculovirus iap repeat (BIR). BIRPs contain 1-3 imperfectly repeated BIRs (Figure 1). Most of the IAP family members also harbor a RING domain at the carboxy terminus. The RING functions as an E3 ubiquitin ligase, which is preceded by ubiquitinactivating enzymes (E1) and ubiquitin-conjugating enzymes (E2) in the cascade of protein ubiquitination (6-9). E3 ubiquitin ligases provide specificity for the transfer of ubiquitin moieties onto the target protein. Therefore, IAP-mediated protein ubiquitination has a pivotal role in the regulation of apoptosis, allowing the IAP to control stability of itself and other proteins (10).

Nonstandard abbreviations used: ASO, antisense oligonucleotide; BIR, baculovirus iap repeat; BIRP, BIR-containing protein; c-IAP, cellular IAP; Hid, head involution defective; IAP, inhibitor of apoptosis; IBM, IAP-binding motif; ML-IAP, melanoma-associated IAP; Rpr, reaper; Smac/DIABLO, second mitochondria-derived activator of caspase/direct IAP-binding protein with low PI; TRAIL, TNF-related apoptosis-inducing ligand.

Conflict of interest: The authors have declared that no conflict of interest exists.

Citation for this article: J. Clin. Invest. 115:2673-2678 (2005).

doi:10.1172/JCI26251.
Survivin is a small $(17 \mathrm{kDa})$ protein composed of a single BIR motif and is similar in structure to the IAPs/BIRPs of yeasts and nematodes (11). Targeted deletion of the murine survivin gene revealed a critical role for this protein in the cell cycle through regulation of the spindle formation during mitosis $(12,13)$, a role that is similar to that found in yeasts and the nematode C. elegans $(14,15)$. Additionally, a number of reports have also implicated survivin in apoptotic inhibition, although the details of this role are not entirely clear (16). Nevertheless, survivin is highly expressed in dividing cells and cancer-derived cell lines and has become a valid target for anticancer drugs, including those that use antisense approaches.

In addition to survivin, much attention has been focused on XIAP, in large part because its antiapoptotic properties have been best demonstrated (17). XIAP is a ubiquitously expressed $56-\mathrm{kDa}$ protein that contains 3 BIRs, as well as a RING domain at the carboxy terminus that has been shown to exhibit E3 ubiquitin ligase activity $(9,18)$. A number of reviews have examined the clinical utility of survivin in more detail (19); therefore, this Review will focus on XIAP.

Ectopic expression of XIAP has been shown to confer protection from a wide range of apoptotic stimuli, and this protection is presumed to be largely mediated by the ability of XIAP to directly bind and enzymatically inhibit key components of the apoptotic machinery, the caspases $(20,21)$. This group of cysteine proteases with a specificity for aspartate residues (reviewed in this issue of the JCI, ref. 22) play essential roles in apoptotic cell death. They are initially produced as inactive zymogens, which are typically activated in a hierarchical sequence in which initiator caspases, such as caspase-8, are activated following, for example, engagement of a death receptor signal. Processed initiator caspases subsequently activate, through cleavage, downstream or effector caspases, such as caspase-3, which then disassemble the cell through the orchestrated proteolysis of essential cellular proteins. XIAP has been shown to directly bind and inhibit caspase-3, caspase-7, and caspase-9, which are important mediators of the apoptotic program $(20,23)$. c-IAP1 and c-IAP2 can also block caspase activity; however, they inhibit caspases 100 - to 1,000-fold less efficiently than XIAP (24).

\section{IAPs in cancer}

Many studies have revealed a circumstantial association of IAPs and neoplasia (25). For example, XIAP levels are elevated in many 
A

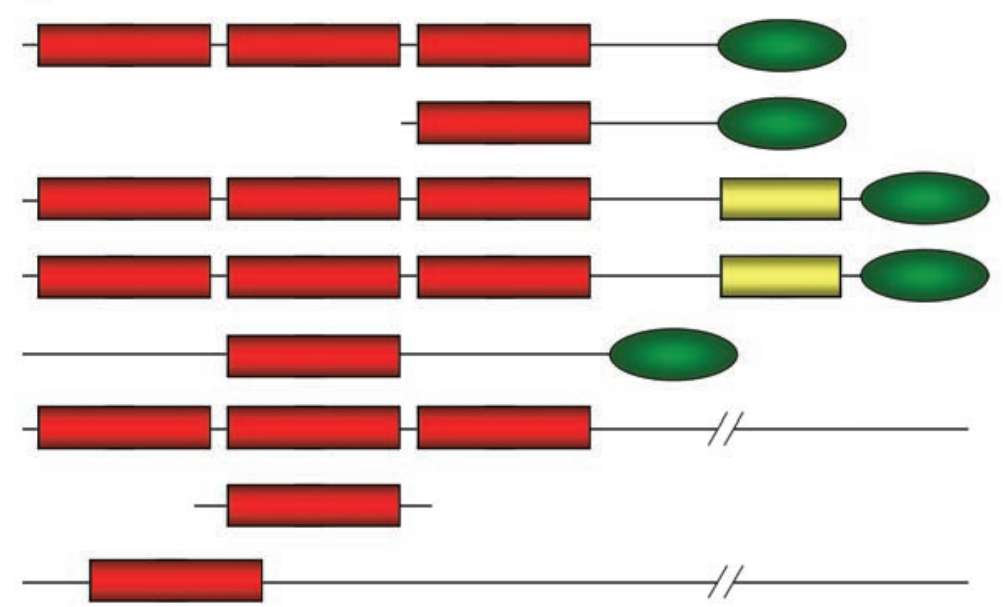

XIAP (hILP-1, MIHA, BIRC4)

hILP-2, TS-IAP

C-IAP1 (HIAP2, MIHB, BIRC2)

C-IAP2 (HIAP1, MIHC, BIRC3)

ML-IAP (Livin, KIAP, BIRC7)

NAIP, BIRC1

Survivin (TIAP, BIRC5)

Apollon (BRUCE, BIRC6)

B
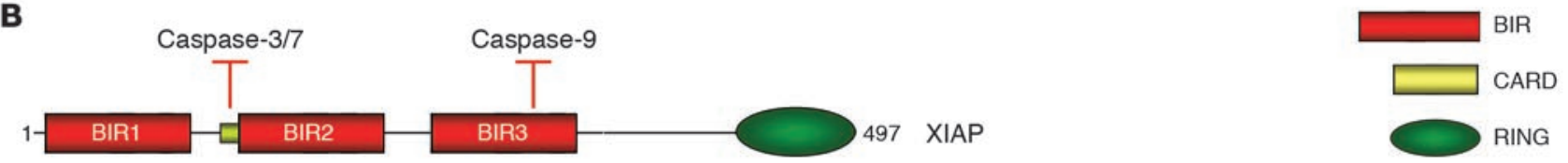

Figure 1

The IAP family members. (A) All IAP members contain 1 or more imperfect baculovirus iap repeats (BIRs), the defining motif of the IAP family. Many of the IAP proteins also posses an E3 ubiquitin ligase RING domain at the carboxy terminus. (B) XIAP can bind and enzymatically inhibit caspase-3, caspase-7, and caspase-9. XIAP binds caspase-3 and caspase-7 at a sequence directly upstream of BIR2, while it binds caspase- 9 in a region of BIR3. CARD, caspase recruitment domain.

cancer cell lines, and several reports have shown that suppression of XIAP protein levels can sensitize cancer cells to chemotherapeutic drugs (26-29). IAP proteins appear to regulate the transcriptional activator NF-кB family, which itself has been associated with malignancy (30-32). NF- $\mathrm{BB}$ activation in turn upregulates expression of c-IAP2, providing a positive feedback loop for cell survival that may be important in the development of some cancers (32). Also, the presence of increased levels of c-IAP2 protein is correlated with carcinogenesis and chemotherapeutic resistance in malignant pleural mesothelioma, a tumor that attacks the pleura of the lung (33). Survivin expression is normally limited to cells of the developing fetus and is not expressed in differentiated adult tissue (11). However, aberrant expression of survivin has been detected in a number of different cancers and lymphomas (11), and expression of a dominant-negative form of survivin induced apoptosis in cancer but not normal cell lines (27). Furthermore, ML-IAP was identified as an IAP that is highly expressed in the majority of melanoma cell lines tested, but undetectable in primary melanocytes (34).

Although heightened expression of IAPs has been reported in and may contribute to the progression of many cancers, it is important to caution that many of these results are correlative in nature. For example, to our knowledge, no solid evidence has been reported to show that XIAP is itself an oncogene, or specifically that mutations, translocations, or amplifications at the XIAP locus have ever been found in natural tumors. This is true for all of the IAP/BIRP family, with the single exception of c-IAP2, which has been found to be translocated in mucosal-associated lymphoid tissue (MALT) lymphoma, where a RING-deleted form of c-IAP2 is fused to the MALT1 protein $(35,36)$. Furthermore, while elevated expression of IAPs has been reported in many cancers, it has also been shown that IAP levels are not always correlated with disease progression or prognosis (37-39). However, this does not invalidate the targeting of, for example, XIAP, for therapeutic intervention, but it does emphasize that the relevance of XIAP as an anticancer target should be scrutinized more rigorously than that of a typical oncogene. It is quite possible that future studies will identify alterations in IAPs as a major causative genetic lesion in specific malignancies, and indeed a recent study reported the presence of the closely linked c-IAP1 and c-IAP2 genes in an amplicon associated with esophageal squamous cell carcinoma (40). Nevertheless, the current model of targeting IAPs, particularly XIAP, relies exclusively on expression differences between malignant and normal cells and presumes that tumor cells have selected for enhanced expression levels in the absence of apparent genetic alterations at the XIAP locus.

\section{Two pathways of caspase activation regulated by the IAP proteins}

Two major apoptotic signaling cascades have been described and are generally referred to as the extrinsic (or receptor-mediated) and intrinsic (or mitochondrial) pathways (41) (Figure 2). The extrinsic pathway transduces an intracellular signal into an apoptotic response and is exemplified by proapoptotic members of the TNF receptor superfamily, such as the TNF-related apoptosis-inducing ligand (TRAIL) receptor and the Fas receptor. Ligand-mediated activation of these receptors results in the binding of adaptor molecules that subsequently recruit and promote the activation of procaspase-8 (42-44). In this scheme, activated caspase-8 is thought to function as an initiator caspase, leading to the subsequent cleavage and activation of effector caspases, such as caspase-3, and thus leading to cell death (Figure 2). 


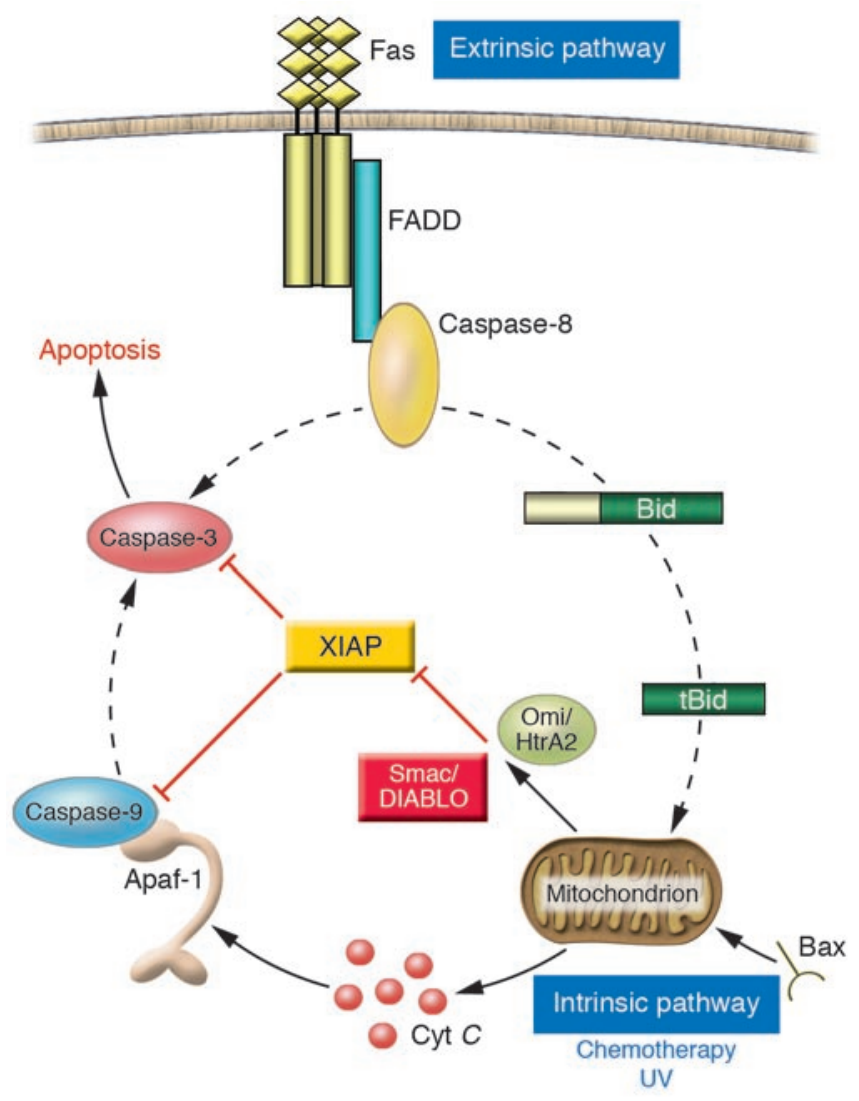

The intrinsic pathway is activated by a range of apoptotic stimuli, including DNA damage, treatment of cells with chemotherapeutic agents, and growth factor withdrawal. The mitochondria serve as the control point for this pathway (45). A pivotal step in this process is the release of cytochrome $c$ from the mitochondria into the cytosol (46). The Bcl-2 family of proteins very carefully regulates this release.

Once released into the cytosol, cytochrome $c$ binds directly to a key cellular component of the apoptotic cascade, apoptotic protease-activating factor-1 (Apaf-1), which subsequently oligomerizes to form a high-molecular weight structure designated the apoptosome (47). This complex is able to recruit and activate caspase-9, which is the key initiator caspase in the intrinsic pathway. Apoptosome-activated caspase-9 is subsequently able to act on downstream effector caspases, such as caspase- 3 and caspase-7, to generate the apoptotic signal (Figure 2).

Signaling crosstalk exists between the intrinsic and extrinsic cell death pathways. For example, the proapoptotic Bcl-2-related protein Bid can be cleaved by caspase- 8 to produce an active form, known as tBid, which can subsequently translocate to mitochondria and lead to the induction of the intrinsic pathway $(48,49)$. Thus, in certain situations, the intrinsic pathway is required for the full induction of receptor-mediated apoptosis and is thought to exert these effects through an amplification loop.

Numerous studies of XIAP have revealed its ability to directly bind to caspase-3, caspase-7, and caspase-9 (50). Consequently, in the scheme of apoptosis shown in Figure 2, XIAP can function directly at the most downstream effector caspase (caspase-3 or caspase-7) in the extrinsic pathway and can block cell death at 2

\section{Figure 2}

$\mathrm{XIAP}$ in apoptosis regulation. Following an apoptotic stimulus, enzymes known as caspases are activated and initiate a cascade leading to the destruction of the cell. The caspases are activated via 2 main avenues, by the stimulation of death receptors (the extrinsic pathway) and by the release of apoptogenic factors from the mitochondria (the intrinsic pathway). XIAP regulates both the extrinsic and the intrinsic apoptotic pathways through direct inhibition of caspase-3 and caspase- 9 .

distinct points (caspase-9 and caspase-3) in the intrinsic pathway. Indeed, biochemical studies have shown that XIAP can be found in the apoptosome (51), presumably in complex with caspase-9, and a number of elegant structural studies have revealed the presence of 2 distinct domains of XIAP that interact with caspase- 3 and caspase-7, and with caspase-9 (52). The caspase-3/caspase-7-binding domain is located directly aminoterminal to the second BIR within XIAP, while the caspase-9-binding domain is contained within the third (most carboxyterminal) BIR (Figure 1B).

\section{IAP antagonists}

After the realization that XIAP can directly bind caspases, the crucial discovery that enforced the concept that XIAP is an attractive therapeutic target came from studies that identified endogenous regulators of its activity. The first, and best, characterized of these is second mitochondria-derived activator of caspase/direct IAP-binding protein with low $\mathrm{pI}$ (Smac/DIABLO), a nuclear-encoded protein that in healthy cells is localized to the mitochondria in a mature form lacking the aminoterminal 55 residues, which are removed during mitochondrial translocation $(53,54)$. Smac/DIABLO is a functional homolog of 3 proapoptotic factors in Drosophila, namely reaper (rpr), head involution defective ( hid), and grim $(55,56)$. The main functional motif of these IAP antagonists is at the extreme amino terminus of Rpr, Hid, and Grim, and the mature form of Smac/DIABLO. Thus, this sequence has been termed the Rpr/Hid/Grim (RHG) motif, or IAP-binding motif (IBM) as a more general term (57). With kinetics that appear to be identical to those of cytochrome $c$, Smac/DIABLO is released from the mitochondria into the cytosol, where it can bind XIAP. Importantly, Smac/DIABLO has been shown to bind precisely into the same 2 grooves within XIAP that can be occupied by caspase- 3 and -7 or caspase-9 (Figure 3), and this leads to an attractive model in which Smac/DIABLO can act as a proapoptotic molecule that functions through the displacement of XIAP from caspases (23).

Subsequent studies in mammalian cells have revealed the existence of additional molecules that target IAPs in a manner akin to that of Smac/DIABLO. Specifically, 2 factors have been described, designated Omi/HtrA2 (58-62) and GSPT1/eRF3 (63), that share with Smac/DIABLO the properties of mitochondrial localization and cytoplasmic release, as well as the presence of an IBM at the amino terminus of the mature protein. Beyond the tetrapeptide sequence, however, there is virtually no similarity between these proteins, and at least in the case of Omi/HtrA2, which appears to function as a chaperone and a regulator of oxidative stress (64), the primary physiological role appears to be very distinct from caspase/XIAP regulation.

\section{Mimetics of IAP antagonists in cancer treatment}

The development of small molecules that mimic Smac/DIABLO and therefore interfere with caspase- 9 binding to XIAP is proving to be a promising avenue for the treatment of cancer $(25,65)$. 

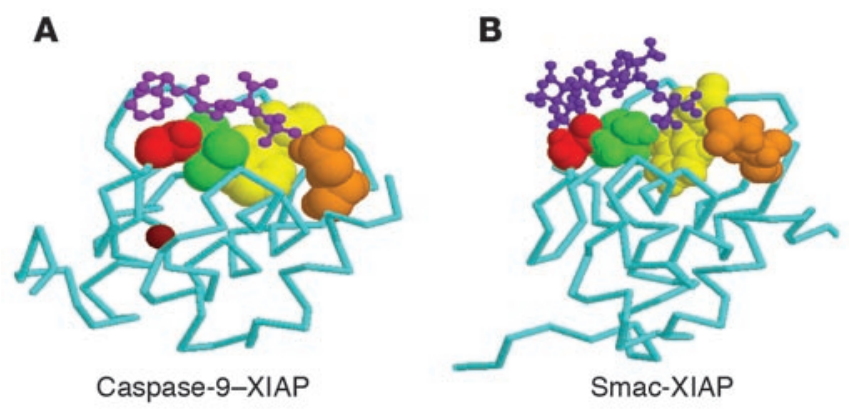

Figure 3

Smac/DIABLO displaces caspase-9 from BIR3 of XIAP. (A) Crystal structure of processed caspase-9 bound to BIR3 of XIAP. The purple peptide represents the first 4 amino acids that contact XIAP, with the amino terminus near the orange residue of XIAP. Coordinates were obtained from Brookhaven Protein Databank file 1NW9 (82). (B) NMR structure of the Smac/DIABLO IBM (purple peptide with the amino terminus near the orange residue of XIAP) bound to the same groove on BIR3 of XIAP that binds caspase-9, thus abrogating the ability of XIAP to block caspase-9 activity. Coordinates were obtained from Brookhaven Protein Databank file 1G3F (83). The 4 residues displayed in van der Waals radii spacefill on XIAP BIR3 are Gly306 (red), Leu307 (green), Trp310 (yellow), and Glu314 (orange). These 4 residues are critical in forming the Smac/DIABLO-binding groove on XIAP BIR3 (84). Images were created using Protein Explorer $(85,86)$.

Furthermore, since the extrinsic and intrinsic pathways are both regulated by XIAP at the step of caspase- 3 activation, small molecules that interfere with this interaction are also being tested. Smac/DIABLO IBM peptides, when used in conjunction with TRAIL, proved to promote apoptosis in cancer cells through caspase- 9 and resulted in complete tumor regression in a murine intracranial malignant glioma xenograft model $(27,66,67)$. However, the use of peptides in therapy is not feasible, because of the proteolytic instability and low cellular permeability of the peptide. Thus, variations on the first 4 residues of Smac/DIABLO have been synthesized to make it a more stable moiety (68-70). These alterations have provided reagents with higher affinity for XIAP than Smac/DIABLO, and lower concentrations of the compounds were required for activity. One of these molecules bound to and antagonized c-IAP1 and c-IAP2, in addition to XIAP, and sensitized HeLa cells to TNF-induced apoptosis (71). This finding suggests that c-IAP1 and c-IAP2 are downstream of NF- KB activity and function to inhibit caspase- 8 activation, allowing prosurvival signals to proceed. Thus Smac/DIABLO mimetics could also provide therapy for inflammation disorders that arise from increased TNF stimulation.

Continual efforts to increase the efficacy of Smac/DIABLO peptide agonists have led other groups to devise small-molecule nonpeptidic compounds that target BIR2 of XIAP, liberating caspase- $3(72,73)$. There may be an advantage to pharmacological agents that antagonize XIAP to release caspase- 3 , since this caspase is at the convergence of both the extrinsic and the intrinsic pathway. The use of these compounds resulted in apoptosis in cancer cells without the requirement for concomitant chemotherapeutic treatment. Furthermore, targeting the release of caspase-3 would eliminate the requirement for mitochondriamediated activation, which is blocked in many cancers by the deregulation of the Bcl-2 pathway (72).

\section{IAP antisense technology in cancer treatment}

Another approach to blocking IAP activity in order to promote caspase activation in cancer cells is to downregulate protein levels by delivering IAP antisense into the cell. Downregulation of XIAP protein levels by adenovirus-mediated introduction of antisense induced apoptosis in chemoresistant ovarian cancer cells (26). Furthermore, a similar antisense approach against c-IAP2 showed c-IAP2 to be a major contributor of chemotherapeutic resistance in pleural mesothelioma (33).

More recently, antisense oligonucleotides (ASOs) have been used in studies to reduce IAP protein levels. ASO technology uses approximately 20 -bp oligonucleotide sequences targeted to the mRNA of the protein of interest; this interferes with expression of the transcript. Reduction of XIAP protein levels in bladder cancer using XIAP ASOs was reported to induce apoptosis and contribute to doxorubicin cytotoxicity (28). Combining ASOs targeted against XIAP and survivin with radiotherapy delayed tumor growth in a mouse lung cancer model and decreased the survival of H460 lung cancer cells (74).

In contrast, a recent study in which ASO technology was used to suppress XIAP levels in cells over time determined that not all chemotherapeutic drugs are effective in combination with decreased XIAP protein levels; this knowledge will prove beneficial in the development of treatment schemes for patients in clinical trials (29). A survivin ASO (ISIS 23722; Isis Pharmaceuticals Inc.) is in preclinical development, and ASOs that target XIAP are in phase I clinical trials (29).

\section{Other functions of IAPs}

It is widely assumed that the primary physiological role of XIAP is as an apoptotic suppressor, and XIAP is often referred to as the only known endogenous caspase inhibitor in mammals. The biochemical evidence for this is compelling, and the caspase-suppressing effects are so striking (with inhibitory constants in the nanomolar range) that the argument has been made that the XIAP-caspase interaction cannot be accidental. It is, however, worth bearing in mind that the biological evidence for XIAP being an essential endogenous inhibitor lags behind the in vitro data. For example, Xiap-deficient mice exhibit no overt apoptotic abnormalities that might be expected (75), although a sensitivity of sympathetic neurons following cytochrome $c$ injection has been reported (76). This is not to say that Xiap-null mice are indistinguishable from control animals: recent reports have revealed alterations in intracellular copper levels (77), and in mammary gland development (78), but these phenotypic differences are unlikely to be caused by alterations in apoptotic sensitivity. The prevailing view is that Xiap-deficient mice survive through a degenerate mechanism, such as compensatory expression of other Iap family members, particularly of c-IAP1, which has been reported in embryonic fibroblasts derived from these animals (75). However, the caspase-inhibitory properties of the c-IAP proteins are much weaker than those of XIAP; this indicates nonredundant roles for these proteins. Other studies from several laboratories have implicated XIAP in a number of cellular functions unrelated to caspases, such as the activation of signal transduction cascades including JNK, NF-кB, TGF- $\beta$, and Akt $(30,31,79,80)$. To date, however, these studies have all relied on ectopic expression of XIAP, and to our knowledge no studies have been described to suggest alterations in these signaling pathways in Xiap-null animals. Thus, the evidence supporting a physiological role for XIAP as a signal transduction intermediate is no stronger than that suggest- 
ing a function in apoptotic regulation. Recently, our laboratory has linked XIAP to copper homeostasis (77), through an interaction with MURR1, a mammalian factor whose gene was identified by positional cloning in an inbred canine strain affected by a non-Wilsonian inherited copper toxicosis disorder (81). MURR1 was found to be a target of ubiquitination by the RING-mediated E3 ubiquitin ligase activity of XIAP, and, consistent with a model in which XIAP can function to regulate MURR1 and copper, tissues from Xiap-null mice were found to contain at the same time higher levels of Murr1 and lower levels of intracellular copper, compared with control animals (77). Thus, clearly much work remains to elucidate the true physiological functions of XIAP. The concept that XIAP participates in oncogenesis and is an anticancer target remains valid if XIAP's participation is due not to caspase inhibition, but to an alternative regulatory function in, for example, intracellular signaling or copper metabolism. The possibility that XIAP is exerting noncaspase-regulatory properties may, however, affect the experimental approaches that need be taken to develop therapeutically effective antagonists of XIAP function.

\section{Conclusions}

The multifunctional IAP proteins are involved in apoptosis regulation, cell signaling, and cell division. Substantial progress has been made in identifying the IAP proteins as factors in cancer development, progression, and desensitization to a wide array of chemotherapeutic drugs. This knowledge should lead to pharma- cological agents that antagonize or downregulate IAP proteins for the treatment of cancer in patients. However, because of the multifunctional nature of the IAP proteins, the consequences of affecting other nonapoptotic processes in the cell need to be considered in the design of preclinical agents for use in clinical trials. Nevertheless, the use of either Smac/DIABLO mimetics or IAP ASOs has resulted in tumor regression in mouse cancer models. Furthermore, ASO technology targeted against other apoptotic regulatory proteins besides IAP proteins has already proven to be a viable avenue of cancer treatment, making the promise of anticancer therapy by targeting of IAP proteins a reality.

\section{Acknowledgments}

We would like to express our gratitude to the Duckett laboratory for helpful discussions, and in particular to John Wilkinson for critical reading of the manuscript. We apologize to those researchers whose work we could not cite because of space constraints. This work was supported in part by NIH grant T32 HL07517 to C.W. Wright and by the University of Michigan Biological Scholars Program, Department of Defense Idea Award PC040215, and NIH grant GM067827 to C.S. Duckett.

Address correspondence to: Colin S. Duckett, Medical Science I, Room 5315, 1301 Catherine Street, Ann Arbor, Michigan 481090602, USA. Phone: (734) 615-6414; Fax: (734) 615-7012; E-mail: colind@umich.edu.
1. Crook, N.E., Clem, R.J., and Miller, L.K. 1993. An apoptosis-inhibiting baculovirus gene with a zinc finger-like motif. J. Virol. 67:2168-2174.

2. Birnbaum, M.J., Clem, R.J., and Miller, L.K. 1994. An apoptosis-inhibiting gene from a nuclear polyhedrosis virus encoding a polypeptide with Cys/ His sequence motifs. J. Virol. 68:2521-2528.

3. Clem, R.J., Fechheimer, M., and Miller, L.K. 1991. Prevention of apoptosis by a baculovirus gene during infection of insect cells. Science. 254:1388-1390.

4. Salvesen, G.S., and Duckett, C.S. 2002. IAP proteins: blocking the road to death's door [review]. Nat. Rev. Mol. Cell Biol. 3:401-410.

5. Uren, A.G., Coulson, E.J., and Vaux, D.L. 1998. Conservation of baculovirus inhibitor of apoptosis repeat proteins (BIRPs) in viruses, nematodes, vertebrates and yeasts. Trends Biochem. Sci. 23:159-162.

6. Huang, H.-K., et al. 2000. The inhibitor of apoptosis, cIAP2, functions as a ubiquitin-protein ligase and promotes in vitro monoubiquitination of caspases 3 and 7. J. Biol. Chem. 275:26661-26664.

7. Olson, M.R., et al. 2003. A GH3-like domain in reaper is required for mitochondrial localization and induction of IAP degradation. J. Biol. Chem. 278:44758-44768.

8. Suzuki, Y., Nakabayashi, Y., and Takahashi, R. 2001. Ubiquitin-protein ligase activity of X-linked inhibitor of apoptosis protein promotes proteasomal degradation of caspase- 3 and enhances its anti-apoptotic effect in Fas-induced cell death. Proc. Natl. Acad. Sci. U. S. A. 98:8662-8667.

9. Yang, Y., Fang, S., Jensen, J.P., Weissman, A.M., and Ashwell, J.D. 2000. Ubiquitin protein ligase activity of IAPs and their degradation in proteasomes in response to apoptotic stimuli. Science. 288:874-877.

10. Jesenberger, V., and Jentsch, S. 2002. Deadly encounter: ubiquitin meets apoptosis. Nat. Rev. Mol. Cell Biol. 3:112-121.

11. Ambrosini, G., Adida, C., and Altieri, D.C. 1997. A novel anti-apoptosis gene, survivin, expressed in cancer and lymphoma. Nat. Med. 3:917-921.
12. Uren, A.G., et al. 2000. Survivin and the inner centromere protein INCENP show similar cell-cycle localization and gene knockout phenotype. Curr. Biol. 10:1319-1328.

13. Okada, H., et al. 2004. Survivin loss in thymocytes triggers p53-mediated growth arrest and p53-independent cell death. J. Exp. Med. 199:399-410.

14. Uren, A.G., et al. 1999. Role for yeast inhibitor of apoptosis (IAP)-like proteins in cell division. Proc. Natl. Acad. Sci. U. S. A. 96:10170-10175.

15. Fraser, A.G., James, C., Evan, G.I., and Hengartner, M.O. 1999. Caenorhabditis elegans inhibitor of apoptosis protein (IAP) homologue BIR-1 plays a conserved role in cytokinesis. Curr. Biol. 9:292-301.

16. Altieri, D.C. 2003. Survivin, versatile modulation of cell division and apoptosis in cancer. Oncogene. 22:8581-8589

17. Holcik, M., Gibson, H., and Korneluk, R.G. 2001. XIAP: apoptotic brake and promising therapeutic target. Apoptosis. 6:253-261.

18. Li, X., Yang, Y., and Ashwell, J.D. 2002. TNF-RII and c-IAP1 mediate ubiquitination and degradation of TRAF2. Nature. 416:345-347.

19. Altieri, D.C. 2004. Molecular circuits of apoptosis regulation and cell division control: the survivin paradigm. J. Cell. Biochem. 92:656-663.

20. Deveraux, Q.L., Takahashi, R., Salvesen, G.S., and Reed, J.C. 1997. X-linked IAP is a direct inhibitor of cell-death proteases. Nature. 388:300-304.

21. Shi, Y. 2004. Caspase activation, inhibition, and reactivation: a mechanistic view. Protein Sci. 13:1979-1987.

22. Lavrik, I.N., Golks, A., and Krammer, P.H. 2005. Caspases: pharmacological manipulation of cell death. J. Clin. Invest. 115:2665-2672. doi:10.1172/ JCI26252.

23. Shiozaki, E.N., and Shi, Y. 2004. Caspases, IAPs and Smac/DIABLO: mechanisms from structural biology. Trends Biochem. Sci. 29:486-494.

24. Roy, N., Deveraux, Q.L., Takahashi, R., Salvesen, G.S., and Reed, J.C. 1997. The c-IAP-1 and c-IAP-2 proteins are direct inhibitors of specific caspases. EMBO J. 16:6914-6925.
25. Schimmer, A.D. 2004. Inhibitor of apoptosis proteins: translating basic knowledge into clinical practice. Cancer Res. 64:7183-7190.

26. Sasaki, H., Sheng, Y., Kotsuji, F., and Tsang, B.K. 2000. Down-regulation of X-linked inhibitor of apoptosis protein induces apoptosis in chemoresistant human ovarian cancer cells. Cancer Res. 60:5659-5666.

27. Yang, L., Cao, Z., Yan, H., and Wood, W.C. 2003. Coexistence of high levels of apoptotic signaling and inhibitor of apoptosis proteins in human tumor cells: implication for cancer specific therapy. Cancer Res. 63:6815-6824.

28. Bilim, V., Kasahara, T., Hara, N., Takahashi, K., and Tomita, Y. 2003. Role of XIAP in the malignant phenotype of transitional cell cancer (TCC) and therapeutic activity of XIAP antisense oligonucleotides against multidrug-resistant TCC in vitro. Int. J. Cancer. 103:29-37.

29. McManus, D.C., et al. 2004. Loss of XIAP protein expression by RNAi and antisense approaches sensitizes cancer cells to functionally diverse chemotherapeutics. Oncogene. 23:8105-8117.

30. Hofer-Warbinek, R., et al. 2000. Activation of NF-кB by XIAP, the X chromosome-linked inhibitor of apoptosis, in endothelial cells involves TAK1. J. Biol. Chem. 275:22064-22068.

31. Birkey Reffey, S., Wurthner, J.U., Parks, W.T., Roberts, A.B., and Duckett, C.S. 2001. X-linked inhibitor of apoptosis protein functions as a cofactor in transforming growth factor- $\beta$ signaling. J. Biol. Chem. 276:26542-26549.

32. Chu, Z.-L., et al. 1997. Suppression of tumor necrosis factor-induced cell death by inhibitor of apoptosis c-IAP2 is under NF-кB control. Proc. Natl. Acad. Sci. U. S. A. 94:10057-10062.

33. Gordon, G.J., et al. 2002. Inhibitor of apoptosis protein-1 promotes tumor cell survival in mesothelioma. Carcinogenesis. 23:1017-1024.

34. Vucic, D., Stennicke, H.R., Pisabarro, M.T., Salvesen, G.S., and Dixit, V.M. 2000. ML-IAP, a novel inhibitor of apoptosis that is preferentially expressed in human melanomas. Curr. Biol. 10:1359-1366. 
35. Dierlamm, J., et al. 1999. The apoptosis inhibitor gene API2 and a novel $18 \mathrm{q}$ gene, $M L T$, are recurrently rearranged in the $\mathrm{t}(11 ; 18)(\mathrm{q} 21 ; \mathrm{q} 21)$ associated with mucosa-associated lymphoid tissue lymphomas. Blood. 93:3601-3609.

36. Uren, A.G., et al. 2000. Identification of paracaspases and metacaspases: two ancient families of caspase-like proteins, one of which plays a key role in MALT lymphoma. Mol. Cell. 6:961-967.

37. Tamm, I., et al. 2000. Expression and prognostic significance of IAP-family genes in human cancers and myeloid leukemias. Clin. Cancer Res. 6:1796-1803.

38. Ferreira, C.G., et al. 2001. Expression of X-linked inhibitor of apoptosis as a novel prognostic marker in radically resected non-small cell lung cancer patients. Clin. Cancer Res. 7:2468-2474.

39. Carter, B.Z., et al. 2003. Caspase-independent cell death in AML: caspase inhibition in vitro with pancaspase inhibitors or in vivo by XIAP or Survivin does not affect cell survival or prognosis. Blood. 102:4179-4186.

40. Imoto, I., et al. 2001. Identification of $c I A P 1$ as a candidate target gene within an amplicon at $11 \mathrm{q} 22$ in esophageal squamous cell carcinomas. Cancer Res. 61:6629-6634.

41. Budihardjo, I., Oliver, H., Lutter, M., Luo, X., and Wang, X. 1999. Biochemical pathways of caspase activation during apoptosis. Annu. Rev. Cell Dev. Biol. 15:269-290.

42. Hsu, H., Shu, H.-B., Pan, M.-G., and Goeddel, D.V. 1996. TRADD-TRAF2 and TRADD-FADD interactions define two distinct TNF receptor 1 signal transduction pathways. Cell. 84:299-308.

43. Kischkel, F.C., et al. 1995. Cytotoxicity-dependent APO-1 (Fas/CD95)-associated proteins form a death-inducing signaling complex (DISC) with the receptor. EMBO J. 14:5579-5588.

44. Boldin, M.P., Goncharov, T.M., Goltsev, Y.V., and Wallach, D. 1996. Involvement of MACH, a novel MORT1/FADD-interacting protease, in Fas/ APO-1- and TNF receptor-induced death. Cell. 85:803-815.

45. Desagher, S., and Martinou, J.C. 2000. Mitochondria as the central control point of apoptosis. Trends Cell Biol. 10:369-377.

46. Li, P., et al. 1997. Cytochrome $c$ and dATP-dependent formation of Apaf-1/caspase- 9 complex initiates an apoptotic protease cascade. Cell. 91:479-489.

47. Zou, H., Henzel, W.J., Liu, X., Lutschg, A., and Wang, X. 1997. Apaf-1, a human protein homologous to C. elegans CED-4, participates in cytochrome $c$-dependent activation of caspase-3. Cell. 90:405-413.

48. Luo, X., Budihardjo, I., Zou, H., Slaughter, C., and Wang, X. 1998. Bid, a $\mathrm{Bcl} 2$ interacting protein, mediates cytochrome $c$ release from mitochondria in response to activation of cell surface death receptors. Cell. 94:481-490.

49. Li, H., Zhu, H., Xu, C.J., and Yuan, J. 1998. Cleavage of BID by caspase 8 mediates the mitochondrial damage in the Fas pathway of apoptosis. Cell. 94:491-501.

50. Salvesen, G.S., and Abrams, J.M. 2004 Caspase activation: stepping on the gas or releasing the brakes? Lessons from humans and flies [review]. Oncogene. 23:2774-2784.

51. Bratton, S.B., Lewis, J., Butterworth, M., Duckett, C.S., and Cohen, G.M. 2002. XIAP inhibition of caspase- 3 preserves its association with the Apaf- 1 apoptosome and prevents CD95- and Bax-induced apoptosis. Cell Death Differ. 9:881-892.

52. Stennicke, H.R., Ryan, C.A., and Salvesen, G.S 2002. Reprieval from execution: the molecular basis of caspase inhibition. Trends Biochem. Sci. 27:94-101.

53. Verhagen,A.M., et al.2000. Identification of DIABLO, a mammalian protein that promotes apoptosis by binding to and antagonizing IAP proteins. Cell. 102:43-53.

54. Du, C., Fang, M., Li, Y., Li, L., and Wang, X. 2000. Smac, a mitochondrial protein that promotes cytochrome c-dependent caspase activation by eliminating IAP inhibition. Cell. 102:33-42.

55. Wu, J.W., Cocina, A.E., Chai, J., Hay, B.A., and Shi, Y. 2001. Structural analysis of a functional DIAP1 fragment bound to grim and hid peptides. Mol. Cell. 8:95-104.

56. Wright, C.W., and Clem, R.J. 2001. Sequence requirements for hid binding and apoptosis regulation in the anti-apoptotic baculovirus protein Op-IAP: hid binds Op-IAP in a manner similar to Smac binding of XIAP. J. Biol. Chem. 277:2454-2462.

57. Shi, Y. 2002. A conserved tetrapeptide motif: potentiating apoptosis through IAP-binding. Cell Death Differ. 9:93-95.

58. Hegde, R., et al. 2001. Identification of Omi/HtrA2 as a mitochondrial apoptotic serine protease that disrupts IAP-caspase interaction. J. Biol. Chem. 277:432-438.

59. Martins, L.M., et al. 2001. The serine protease Omi/HtrA2 regulates apoptosis by binding XIAP through a Reaper-like motif. J. Biol. Chem. 277:439-444

60. Suzuki, Y., et al. 2001. A serine protease, HtrA2, is released from the mitochondria and interacts with XIAP, inducing cell death. Mol. Cell. 8:613-621.

61. van Loo, G., et al. 2002. The serine protease Omi/ HtrA2 is released from mitochondria during apoptosis. Omi interacts with caspase-inhibitor XIAP and induces enhanced caspase activity. Cell Death Differ. 9:20-26.

62. Vaux, D.L., and Silke, J. 2003. Mammalian mitochondrial IAP binding proteins. Biochem. Biophys. Res. Commun. 304:499-504.

63. Hegde, R., et al. 2003. The polypeptide chainreleasing factor GSPT1/eRF3 is proteolytically processed into an IAP-binding protein. J. Biol. Chem. 278:38699-38706

64. Martins, L.M., et al. 2004. Neuroprotective role of the reaper-related serine protease $\mathrm{HtrA} 2 / \mathrm{Omi}$ revealed by targeted deletion in mice. $\mathrm{Mol}$. Cell. Biol. 24:9848-9862.

65. Huang, Y., Lu, M., and Wu, H. 2004. Antagonizing XIAP-mediated caspase-3 inhibition. Achilles' heel of cancers? [review]. Cancer Cell. 5:1-2.

66. Fulda, S., Wick, W., Weller, M., and Debatin, K.M 2002. Smac agonists sensitize for Apo2L/TRAILor anticancer drug-induced apoptosis and induce regression of malignant glioma in vivo. Nat. Med. 8:808-815.

67. Arnt, C.R., Chiorean, M.V., Heldebrant, M.P., Gores, G.J., and Kaufmann, S.H. 2002. Synthetic Smac/ DIABLO peptides enhance the effects of chemotherapeutic agents by binding XIAP and cIAP1 in situ. J. Biol. Chem. 277:44236-44243.

68. Li, C.J., Friedman, D.J., Wang, C., Metelev, V., and
Pardee, A.B. 1995. Induction of apoptosis in uninfected lymphocytes by HIV-1 Tat protein. Science. 268:429-431.

69. Sun, H., et al. 2004. Structure-based design, synthesis, and evaluation of conformationally constrained mimetics of the second mitochondria-derived activator of caspase that target the $\mathrm{X}$-linked inhibitor of apoptosis protein/caspase-9 interaction site. J. Med. Chem. 47:4147-4150.

70. Oost, T.K., et al. 2004. Discovery of potent antagonists of the antiapoptotic protein XIAP for the treatment of cancer. J. Med. Chem. 47:4417-4426.

71. Li, L., et al. 2004. A small molecule Smac mimic potentiates TRAIL- and TNFalpha-mediated cell death. Science. 305:1471-1474.

72. Wu, T.Y., Wagner, K.W., Bursulaya, B., Schultz, P.G., and Deveraux, Q.L. 2003. Development and characterization of nonpeptidic small molecule inhibitors of the XIAP/caspase-3 interaction. Chem. Biol. 10:759-767.

73. Schimmer, A.D., et al. 2004. Small-molecule antagonists of apoptosis suppressor XIAP exhibit broad antitumor activity. Cancer Cell. 5:25-35.

74. Cao, C., Mu, Y., Hallahan, D.E., and Lu, B. 2004. $\mathrm{XIAP}$ and survivin as therapeutic targets for radiation sensitization in preclinical models of lung cancer. Oncogene. 23:7047-7052.

75. Harlin, H., Reffey, S.B., Duckett, C.S., Lindsten, T., and Thompson, C.B. 2001. Characterization of XIAP-deficient mice. Mol. Cell. Biol. 21:3604-3608.

76. Potts, P.R., Singh, S., Knezek, M., Thompson, C.B., and Deshmukh, M. 2003. Critical function of endogenous XIAP in regulating caspase activation during sympathetic neuronal apoptosis. J. Cell Biol. 163:789-799.

77. Burstein, E., et al. 2004. A novel role for XIAP in copper homeostasis through regulation of MURR1. EMBO J. 23:244-254.

78. Olayioye, M.A., et al. 2005. XIAP-deficiency leads to delayed lobuloalveolar development in the mammary gland. Cell Death Differ. 12:87-90.

79. Sanna, M.G., Duckett, C.S., Richter, B.W.M., Thompson, C.B., and Ulevitch, R.J. 1998. Selective activation of JNK1 is necessary for the anti-apoptotic activity of hILP. Proc. Natl. Acad. Sci. U. S. A. 95:6015-6020.

80. Asselin, E., Mills, G.B., and Tsang, B.K. 2001. XIAP regulates Akt activity and caspase-3-dependent cleavage during cisplatin-induced apoptosis in human ovarian epithelial cancer cells. Cancer Res. 61:1862-1868.

81. van De Sluis, B., Rothuizen, J., Pearson, P.L., van Oost, B.A., and Wijmenga, C. 2002. Identification of a new copper metabolism gene by positional cloning in a purebred dog population. Hum. Mol. Genet. 11:165-173.

82. Shiozaki, E.N., et al. 2003. Mechanism of XIAPmediated inhibition of caspase-9. Mol. Cell. 11:519-527.

83. Liu, Z., et al. 2000. Structural basis for binding of Smac/DIABLO to the XIAP BIR3 domain. Nature. 408:1004-1008.

84. Wu, G., et al. 2000. Structural basis of IAP recognition by Smac/DIABLO. Nature. 408:1008-1012.

85. Martz, E. 2002. Protein explorer: easy yet powerful macromolecular visualization. Trends Biochem. Sci. 27:107-109.

86. Protein Explorer. http://proteinexplorer.org. 